\title{
Research on Food Inventory-Distribution Integrated Optimization for VMI Systems
}

\author{
Qi Dong a), Xifu Wang and Yongwang Liu \\ School of Traffic and Transportation, Beijing Jiao tong University, Beijing 100044, China. \\ a) Corresponding author: 17120763@bjtu.edu.cn
}

\begin{abstract}
In this paper, the food inventory-distribution integrated optimization problem on a two-echelon logistics system composed of a single distribution center and many sales points is studied under the conditions of VMI strategy and determinate demand. Considering both inventory cost and distribution cost in logistics system, the author constructs an integrated optimization model to minimize the system cost and puts forward the iterative algorithm to solve the model, so as to get the optimal solution of inventory control and distribution decision-making. At the same time, distribution is divided into TL and LTL to consider in the calculation of the distribution cost. Finally, this paper selects an enterprise logistics system as an example and uses mathematical simulation MATLAB software to solve the problem, which demonstrates that the optimization model and algorithm is effective and reasonable.
\end{abstract}

Key words: Vendor Managed Inventory, Inventory and Distribution, Integrated Optimization.

\section{INTRODUCTION}

With the rapid development of social economy and increasingly fierce market competition, customers are increasingly demanding for goods and services, and customer-centric corporate logistics management is facing a more complex competitive environment. As a more mature management model, supply chain management aims to increase the core competitiveness of individual enterprises and at the same time emphasizes the strategic cooperation among multiple companies. Vendor Managed Inventory (VMI) is a new type of inventory management model based on supply chain management concepts. It breaks the traditional mode of mutual management and mutual inconsistency among companies and manages inventory with systematic ideas. To enable companies in the supply chain to operate synchronously and enhance their market responsiveness and competitiveness.

The foreign scholars' research results in this area are relatively abundant. They have expanded from single-cycle to multi-cycle and try to expand the food integrated optimization of inventory and transportation to the strategic level and serve the long-term strategic planning of enterprises. This paper will consider the integrated optimization of inventory and distribution under the VMI model on the basis of their ideas.

\section{PROBLEM ANALYSIS}

In the VMI model, the food suppliers have the right to share customers' sales and inventory information, replenish stocks based on customer stock and sales forecasts, and formulate inventory and distribution plans in an integrated manner, so it will pay more attention to the coordination between inventory and distribution. As the two important elements of the logistics system, there is an inverse relationship between inventory and Distribution: when pursuing smaller inventory costs, you need to reduce inventory, which leads to a reduction in the amount of each delivery then the number of delivery must be increased, so the cost of distribution will increase; If you are pursuing lower distribution costs, you will need to increase the delivery volume of each delivery and reduce the number of delivery. This will inevitably lead to an increase in inventory and an increase in inventory costs. Therefore, how to 
ensure the quality of logistics services, realize the integrated optimization of inventory and distribution in logistics systems, effectively balance the contradiction between inventory and distribution, and effectively reduce logistics costs are important issues that the food suppliers need to solve under the VMI model.

This article is based on the integrated optimization of inventory and distribution under the VMI model. Considering a secondary logistics system consisting of one supplier and multiple customers, the supplier has a temporary delivery center in a certain area. However, the distribution center does not have the inventory function and is only responsible for relaying the goods supplied by the factory to customers in the region. The supplier distributes a variety of goods for each customer at the same time and is responsible for managing the inventory of each customer in the area. After the goods are delivered to the distribution center, temporary storage, sorting, picking, and other operations are performed and distributed to each customer in the area in time.

\section{Model construction}

The integrated optimization of inventory and distribution is from the system point of view, in order to achieve the minimum cost of the entire system, the inventory control and distribution decision-making in logistics are considered as a whole to study, achieve the balance between inventory costs and distribution costs. The system cost consists of inventory cost and delivery cost. The inventory cost includes the ordering cost, the inventory holding cost and the out-of-stock cost. The distribution cost includes the distribution transportation cost, the loading and unloading handling cost, the packaging cost, the sorting and distribution cost, and the circulation processing cost. From this it can be seen that determining the frequency of delivery, the volume of distribution, as well as the allocation of transport vehicles and route optimization solutions are the keys to develop the structure.

\section{Symbol Description}

The definitions of the symbols appearing in the model are as follows:

$N$ Number of clients, $i=1,2,3, \ldots, N$ represents each customer, $i=0$ represents the distribution center;

$L$ Types of goods, $l=1,2,3, \ldots, L$ indicates all kinds of goods;

$D_{i l}$ The customer $i$ 's demand for goods $l$ during the planning period;

$D_{\mathrm{T}}$ Total system demand during the planning period, $D_{\mathrm{T}}=\sum_{i=1}^{N} \sum_{l=1}^{L} D_{i l} ;$

$Q$ One delivery of the entire system;

$Q_{i}$ One delivery to customer;

$Q_{i l}$ The number of products to the customer each delivery;

$S_{c}$ The fixed cost of each delivery from the factory to the distribution center;

$S_{p}$ The fixed cost of each distribution center;

$c_{p}$ Unit cost of transportation from factory to distribution center;

$c$ Cost per unit of mileage for transportation journey (not related to load);

$d_{i j}$ Mileage from Customer $i$ to Customer $j$, when $i=0$ indicates the distance from the distribution center to each customer;

$r$ Launch cost of delivery vehicle;

$h_{i l}$ The unit inventory cost of customer for product during the planning period;

$W$ Distribution vehicle maximum load;

$T C_{1}(Q)$ Transfer costs from factory to distribution center;

$T C_{2}(Q)$ Inventory holding costs;

$T C_{3}(Q)$ Distribution costs;

$P(Q)$ Distribution and transportation costs in the distribution process; 
$T C(Q)$ Total system cost during the planning period.

\section{Model Assumption}

The integrated optimization of inventory and distribution is a complex issue. When establishing its mathematical model, many factors and constraints need to be considered. In order to facilitate the establishment of models, this paper proposes the following assumptions:

(1) The planning period of the entire system is fixed, such as one month or two months;

(2) The whole system deals with $L$ kinds of commodities. Suppliers can provide multiple commodities at one time, and the same number of various commodities have the same transportation costs;

(3) The demand rate of each customer for each commodity may be different, but the demand rate can be accurately predicted and will not change during the plan period;

(4) Customers don't allow the out-of-stock phenomenon, that is, setting the stock-out cost to infinity;

(5) The coordinate position of the distribution center and the customer is known and fixed;

(6) The distribution center doesn't have the inventory function. It is only responsible for relaying and distributing the commodities that the supplier needs to deliver to customers in the area;

(7) The distribution center adopts the integrated distribution method, and the vehicle can deliver more than one customer point at the same time;

(8) All merchandise can be mixed. Only the vehicle capacity limit is considered when loading and the maximum load of all delivery vehicles is the same.

Based on the above assumptions, consider the fixed investment costs, transportation costs, storage costs, loading and unloading handling costs, and build a mathematical model as follows:

The constraints of the logistics network node optimization model are as follows:

$$
\min T C(Q)=T C_{1}(Q)+T C_{2}(Q)+T C_{3}(Q)
$$

In Eq. (1) the transfer cost from the factory to the distribution center includes the fixed cost for each shipment of the factory and the transportation cost of the goods from the factory to the distribution center, that is:

$$
T C_{1}(Q)=\frac{D_{\mathrm{T}}}{Q} \times S_{c}+D_{\mathrm{T}} \times c_{p}
$$

Assuming that the demand for goods at each customer during the plan period is stable, When the delivery quantity is, the inventory holding cost at each customer can be approximated as:

$$
T C_{2}(Q)=\frac{1}{2} \sum_{i=1}^{N} \sum_{l=1}^{L} h_{i l} Q_{i l}=\frac{1}{2} \sum_{i=1}^{N} \sum_{l=1}^{L} h_{i l}\left(\frac{Q}{D_{\mathrm{T}}} D_{i l}\right)=\frac{1}{2} \frac{Q}{D_{\mathrm{T}}} \sum_{i=1}^{N} \sum_{l=1}^{L} h_{i l} D_{i l}
$$

The distribution costs are relatively complex. In order to facilitate the understanding of the model, this paper divides it into two parts: the fixed cost of each distribution and the distribution transportation cost of the distribution center to each customer to complete the distribution operation, which is:

$$
T C_{3}(Q)=\frac{D_{\mathrm{T}}}{Q} \times S_{p}+\frac{D_{\mathrm{T}}}{Q} \times P(Q)
$$

Substituting the above eq. (2), eq. (3) and eq. (4) into eq. (1) yields:

$$
\min T C(Q)=\frac{D_{\mathrm{T}}}{Q} \times S_{c}+D_{\mathrm{T}} \times c_{p}+\frac{1}{2} \frac{Q}{D_{\mathrm{T}}} \sum_{i=1}^{N} \sum_{l=1}^{L} h_{i l} D_{i l}+\frac{D_{\mathrm{T}}}{Q} \times S_{p}+\frac{D_{\mathrm{T}}}{Q} \times P(Q)
$$




$$
=\frac{D_{\mathrm{T}}}{Q} \times\left(S_{c}+S_{p}\right)+\frac{1}{2} \frac{Q}{D_{\mathrm{T}}} \sum_{i=1}^{N} \sum_{l=1}^{L} h_{i l} D_{i l}+D_{\mathrm{T}} \times c_{p}+\frac{D_{\mathrm{T}}}{Q} \times P(Q)
$$

In Eq. (5), $P(Q)$ is to select the distribution and transportation costs corresponding to different delivery routes under a certain delivery volume. These costs are related to the number of delivery vehicles and the distribution route. Therefore, it is necessary to identify the number of vehicles required for distribution and delivery routes to calculate the distribution and transportation costs.

Considering that when the distribution center distributes the goods to each customer, the delivery volume at the customer $i$ may exceed the maximum load $W$ of the vehicle, the distribution center uses two methods for each customer's delivery.

(1) Vehicle distribution: Vehicles are fully loaded from the distribution center for individual customers;

(2) LTL distribution: Vehicles are delivered from a distribution center to multiple customers, and each customer's delivery volume is less than the vehicle's capacity;

When the distribution center Create the distribution plan, it should find out the customers whose delivery volume is greater than $W$ first. Assuming that the customer $i$ 's delivery volume is $Q_{i}=n_{i} \times W+q_{i}$ (in which $n_{i}$ is a nonnegative integer, $q_{i}<W$ ), arrange $n_{i}$ vehicle to distribute the whole vehicle first, and the transportation costs of all vehicle distribution are recorded $P_{1}(Q)$. Secondly, the remaining $q_{i}$ products and other customers' demand products are carried out in LTL distribution and all LTL shipping costs are recorded as $P_{2}(Q)$ :

$$
P(Q)=P_{1}(Q)+P_{2}(Q)
$$

Assuming the delivery volume of the customer $i$ is $Q_{i}=n_{i} \times W+q_{i}$ :

$$
P_{1}(Q)=\sum_{i=1}^{N} n_{i}\left(r+2 c d_{0 i}\right)
$$

From the eq. (7), it can be seen that $P_{1}(Q)$ related only to the delivery volume $Q_{i}$ required by each customer, and it is determined when $Q_{i}$ is fixed. All LTL distribution transportation costs can be solved with the following model:

$$
\begin{gathered}
\min P_{2}(Q)=c \sum_{k=1}^{K} \sum_{i=0}^{N} \sum_{j=0}^{N} x_{i j k} d_{i j}+r \sum_{k=1}^{K} \sum_{j=0}^{N} x_{0 j k} \\
\text { S.t. } \sum_{i=1}^{N} q_{i} y_{i k} \leq W \quad k=1,2, \ldots, K \\
\sum_{k=1}^{K} y_{i k}=1 \quad i, j=1,2, \ldots, N ; k=1,2, \ldots, K \\
\sum_{j=1}^{N} x_{0 j k}=1 \quad k=1,2, \ldots, K
\end{gathered}
$$




$$
\begin{aligned}
& \sum_{j=1}^{N} x_{j 0 k}=1 \quad k=1,2, \ldots, K \\
& \sum_{k=1}^{K} x_{i j k}=\sum_{k=1}^{K} x_{j i k}=y_{i k} \quad i, j=1,2, \ldots, N ; k=1,2, \ldots, K \\
& \sum_{i=0}^{N} x_{i j k}=y_{j k} \quad i, j=1,2, \ldots, N ; k=1,2, \ldots, K \\
& x_{i j k}=\left\{\begin{array}{l}
1, \text { if vehicles } k \text { access client } i \text { then visit client } j \\
0, \text { other }
\end{array}\right. \\
& y_{i k}= \begin{cases}1, & \text { if vehicle } \mathrm{k} \text { access clienti } \\
0, & \text { other }\end{cases}
\end{aligned}
$$

The eq. (9) guarantees that the load of each vehicle doesn't exceed the vehicle's specified maximum load, and the eq. (10) indicates that the LTL distribution of each customer has only one vehicle to complete. Eq. (11) and Eq. (12) indicate that each distribution vehicle starts from the distribution center and returns to the distribution center after completing the distribution task. Eq. (13) indicates that each customer has a vehicle passing through, Eq. (14) indicates that if the customer $j$ is delivered by the $k$ vehicle, the vehicle must be from the customer $i$ to the customer $j$, and the eq. (15) and (16) are two decision variables.

In summary, the integrated optimization model of inventory and distribution based on VMI consists of two parts, one is eq. (5), the goal is to calculate the optimal delivery volume and total system cost, and the other is eq. (6), Eq. (7) and Eq. (8), the goal is to calculate the distribution transportation cost in eq. (5) and determine the number of vehicles required for distribution and the distribution route. The analysis is based on the integrated optimization model of inventory and distribution of VMI. Its goal is to minimize the total cost of the entire logistics system, namely:

$$
\min T C(Q)=\frac{D_{\mathrm{T}}}{Q} \times\left(S_{c}+S_{p}\right)+\frac{1}{2} \frac{Q}{D_{\mathrm{T}}} \sum_{i=1}^{N} \sum_{l=1}^{L} h_{i l} D_{i l}+D_{\mathrm{T}} \times c_{p}+\frac{D_{\mathrm{T}}}{Q} \times P(Q)
$$

\section{ANALYSIS OF EXAMPLES}

\section{Case Study Background}

The paper selects a food company which has a distribution center in the sales area as the background. The factory supplies to the distribution center directly and distributes it to various customers through the distribution center. The paper doesn't consider the distribution function of the distribution center, the company's logistics system structure is shown in Figure 1. 


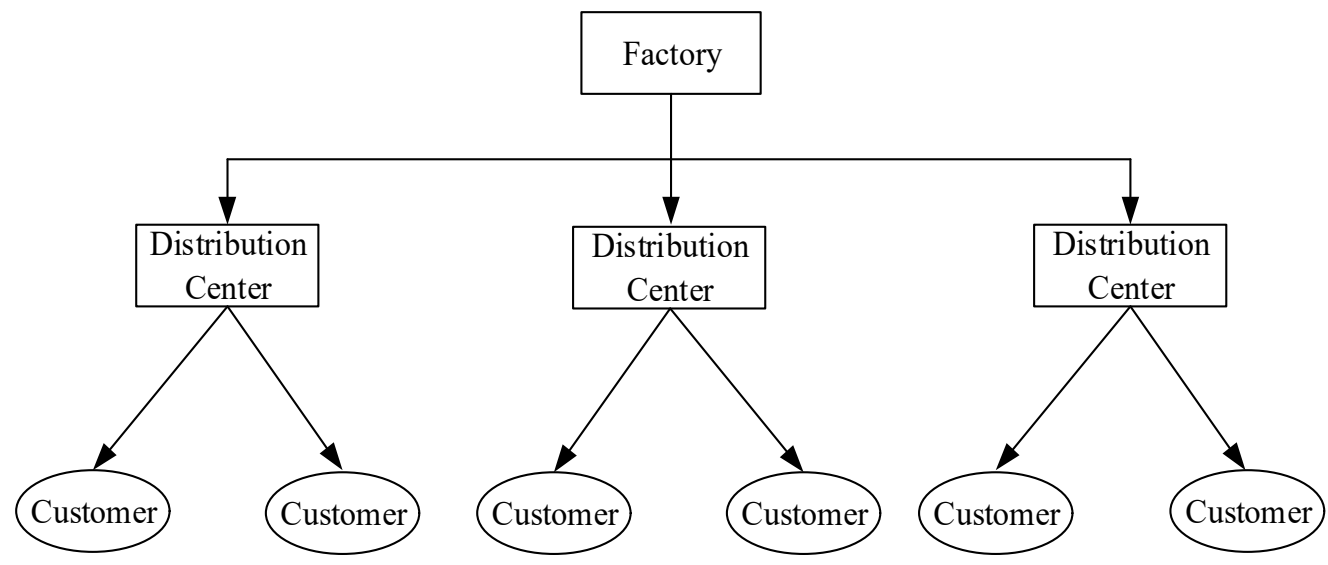

FIGURE 1. Logistics system structure.

\section{Data Management}

The paper selects 21 stable customers of the company in City $\mathrm{M}$ to build a calculation example. The supplier sends the goods from the factory to the distribution center in City M, and then the distribution center sends the customer to each customer through a simple process. The location of the distribution center and each customer is shown in Figure 2.

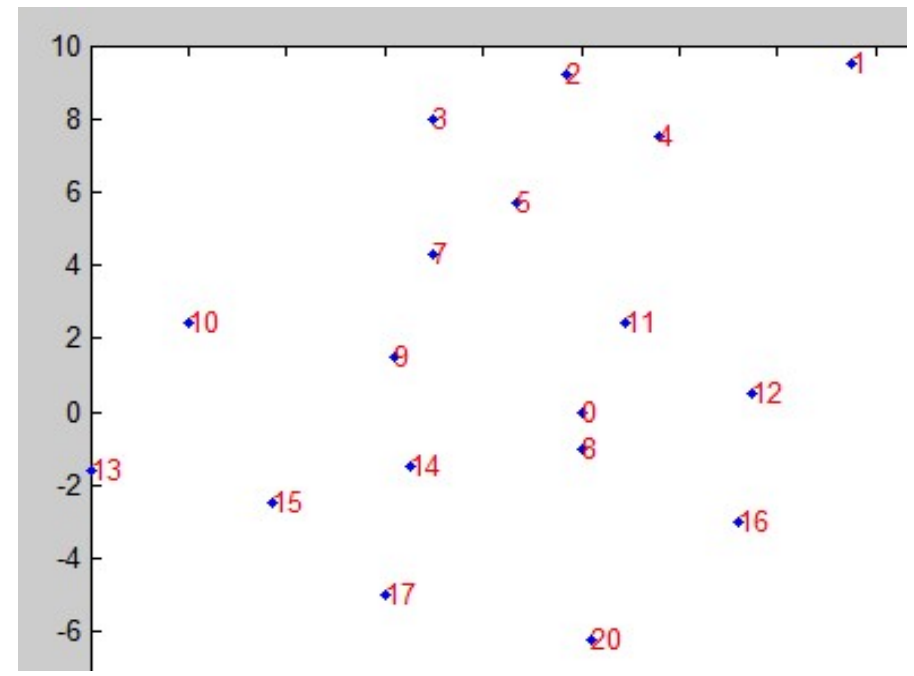

FIGURE 2. Location of distribution center and customers

The coordinates of the distribution center are set to $(0.0,0.0)$, and the addresses and specific coordinates of the distribution center and each customer are shown in Table 1. Other calculation parameters are as follows:

In a certain plan period (one month), the three products $\mathrm{A}, \mathrm{B}$, and $\mathrm{C}$ of the company are selected as delivery products to carry out an optimized simulation analysis of inventory and distribution. The three products are uniformly packaged and transported using the company's standard box $(53 \mathrm{~cm} \times 23 \mathrm{~cm} \times 29 \mathrm{~cm})$. The customer's demand for various commodities during the planning period is shown in Table 2. 
TABLE 1. Location of distribution center and customers

\begin{tabular}{lc}
\hline Numbering & coordinate \\
\hline Customer0 & $(0.0,0.0)$ \\
Customer1 & $(5.5,9.5)$ \\
Customer2 & $(-0.3,9.2)$ \\
Customer3 & $(-3.0,8.0)$ \\
Customer5 & $(1.6,7.5)$ \\
Customer6 & $(-1.3,5.7)$ \\
Customer7 & $(6.8,5.0)$ \\
Customer8 & $(-3.0,4.3)$ \\
Customer9 & $(0.0,-1.0)$ \\
Customer10 & $(-3.8,1.5)$ \\
Customer11 & $(-8.0,2.4)$ \\
Customer12 & $(0.9,2.4)$ \\
Customer13 & $(3.5,0.5)$ \\
Customer14 & $(-10.0,-1.6)$ \\
Customer15 & $(-3.5,-1.5)$ \\
Customer16 & $(-6.3,-2.5)$ \\
Customer17 & $(3.2,-3.0)$ \\
Customer18 & $(-4.0,-5.0)$ \\
Customer19 & $(9.0,-2.6)$ \\
Customer20 & $(-8.8,-8.8)$ \\
Customer21 & $(0.2,-6.2)$ \\
&
\end{tabular}

TABLE 2. Demands of each customer

\begin{tabular}{cccc}
\hline Delivery Volume & Product A & Product B & Product C \\
\hline Customer1 & 60 & 40 & 28 \\
Customer2 & 90 & 30 & 62 \\
Customer3 & 50 & 50 & 0 \\
Customer4 & 40 & 40 & 66 \\
Customer5 & 72 & 48 & 32 \\
Customer6 & 50 & 50 & 46 \\
Customer7 & 30 & 30 & 48 \\
Customer8 & 50 & 67 & 33 \\
Customer9 & 33 & 50 & 36 \\
Customer10 & 30 & 30 & 50 \\
Customer11 & 70 & 70 & 38 \\
Customer12 & 40 & 30 & 60 \\
Customer13 & 120 & 80 & 0 \\
Customer14 & 40 & 43 & 44 \\
Customer15 & 60 & 0 & 52 \\
Customer16 & 62 & 48 & 64 \\
Customer17 & 44 & 36 & 27 \\
Customer18 & 30 & 45 & 45 \\
Customer19 & 0 & 52 & 45 \\
Customer20 & 120 & 55 & 32 \\
\hline
\end{tabular}

Other calculation parameters are as follows:

(1) The fixed cost for each delivery from the factory to the distribution center is 150 yuan.

(2) The transportation cost from the factory to the distribution center is 1 yuan/carton.

(3) The fixed cost for each delivery of the distribution center is 100 yuan.

(4) The unit mileage transportation cost between the distribution center and each customer is 1 yuan $/ \mathrm{km}$. 
Considering that the vehicles in actual distribution will not travel along the straight-line distance between customers, so, in this article, the unit mileage transportation fee is converted into $1.8 \mathrm{yuan} / \mathrm{km}$.

(5) The fixed cost for each distribution route, that is, the fixed start cost of each vehicle is 100 yuan.

(6) The maximum load per vehicle is 50 boxes.

(7) Since the storage requirements of various products in this calculation example are the same, the cost of product storage for each customer during the entire planning period is set to 60 yuan.

\section{Calculation Process}

Based on the model's solution idea and algorithm design, the results obtained using MATLAB7.8, including the minimum total cost of inventory and distribution, the optimal delivery quantity of the goods and the delivery route.

The cost of distribution transportation is solved through iterative method. The results are shown in Table 3.

TABLE 3. Results of calculation

\begin{tabular}{ccccc}
\hline Number of Calculations & Number of Cycles & Single Delivery Volume & Delivery Times & Total Cost \\
\hline 1 & 6 & 231.2490 & 12.6401 & 20063 \\
2 & 6 & 231.5625 & 12.6229 & 20077 \\
3 & 6 & 231.9556 & 12.6015 & 20093 \\
4 & 6 & 231.3716 & 12.6334 & 20061 \\
5 & 6 & 231.1230 & 12.6469 & 20065 \\
6 & 6 & 231.0610 & 12.6503 & 20066 \\
7 & 6 & 231.1230 & 12.6469 & 20065 \\
8 & 6 & 231.1230 & 12.6469 & 20065 \\
9 & 6 & 231.5591 & 12.6431 & 20059 \\
10 & 6 & 231.6503 & 12.6503 & 20066 \\
\hline
\end{tabular}

According to Table 3, the result of each calculation is relatively stable. For this calculation example, the minimum total cost of the logistics system is $T C(Q)=20059$ yuan, and the optimal delivery volume is $Q^{*}=231.5591$ boxes, corresponding to the delivery volume of each product for each customer as shown in Table 4 .

TABLE 4. Distribution Quantity of Each Customer

\begin{tabular}{|c|c|c|c|}
\hline Delivery Volume & Product A & Product B & Product $\mathrm{C}$ \\
\hline Customer1 & 4.7532 & 3.1688 & 2.2182 \\
\hline Customer2 & 7.1298 & 2.3766 & 4.9116 \\
\hline Customer3 & 3.9610 & 3.9610 & 3.9610 \\
\hline Customer4 & 3.1688 & 3.1688 & 0 \\
\hline Customer5 & 5.7038 & 3.8025 & 5.2285 \\
\hline Customer6 & 3.9610 & 3.9610 & 2.5350 \\
\hline Customer7 & 2.3766 & 2.3766 & 3.6441 \\
\hline Customer8 & 3.9610 & 5.3077 & 3.8025 \\
\hline Customer9 & 2.6142 & 3.9610 & 2.6142 \\
\hline Customer10 & 2.3766 & 2.3766 & 3.6441 \\
\hline Customer11 & 5.5454 & 5.5454 & 3.9610 \\
\hline Customer12 & 3.1688 & 2.3766 & 3.0103 \\
\hline Customer13 & 9.5064 & 6.3376 & 4.7532 \\
\hline Customer14 & 3.1688 & 3.4064 & 0 \\
\hline Customer15 & 4.7532 & 0 & 3.4857 \\
\hline Customer16 & 4.9117 & 3.8025 & 4.1194 \\
\hline Customer17 & 3.4857 & 2.8519 & 5.0701 \\
\hline Customer18 & 2.3766 & 3.5649 & 2.1389 \\
\hline Customer19 & 0 & 4.1194 & 3.5649 \\
\hline Customer20 & 9.5064 & 4.3571 & 3.5649 \\
\hline Customer21 & 3.8026 & 2.5350 & 2.5350 \\
\hline
\end{tabular}

The corresponding delivery path is arranged as follows: 
Path 1: Distribution Center - Customer 8 - Customer 13 - Customer 11 - Distribution Center;

Path 2: Distribution Center - Customer 10 - Customer 9 - Customer 18 - Customer 1 - Customer 2 - Distribution Center;

Path 3: Distribution Center - Customer 3 - Customer 14 - Customer 21 - Customer 20 - Distribution Center;

Path 4: Distribution Center - Customer 17 - Customer 19 - Customer 7 - Customer 5 - Distribution Center;

Path 5: Distribution Center - Customer 4 - Customer 6 - Customer 12 - Customer 16 - Customer 15 - Distribution Center.

\section{Results Analysis}

In order to compare with the above calculation results, the paper calculates the individual decision-making situation of inventory and delivery under the condition that the parameter settings are not changed. The calculation results of the two schemes are summarized in Table 5.

TABLE 5. Comparison of Results between Two Schemes

\begin{tabular}{ccc}
\hline Independent Inventory and Distribution Decisions & Integrated Optimization of Inventory and Distribution \\
\hline Single delivery volume & 120.8925 & 231.5591 \\
Delivery route number & 3 & 5 \\
Distribution costs & 11875 & 8291 \\
Inventory costs & 10177 & 11768 \\
Total cost of logistics system & 22052 & 20059 \\
\hline
\end{tabular}

From Table 5, it can be seen that the results of the integrated optimization of inventory and distribution and the individual decision of inventory and distribution in the example differ significantly. The implementation of the integrated optimization of inventory and delivery, although the inventory cost has increased by 1591 yuan, the delivery cost was reduced by 3584 yuan, which reduced the total cost of the logistics system by 1993 yuan. This example is only selected by the 21 customers of the company in the $\mathrm{M}$ city to calculate and the scale is relatively small, the implementation of the integrated optimization of inventory and distribution than the separate consideration of the cost savings of $9.04 \%$, if the scale increases, the cost savings will be more substantial.

\section{CONCLUSION}

Based on the modern logistics integrated management idea, the paper integrates the two important issues of inventory and delivery in logistics system, establishes an integrated optimization mathematical model of inventory and distribution based on VMI, solves the problem through algorithm design and programming and verify the correctness of the model through examples.

The limitation of the research in this paper is that the model is assumed to have no inventory at the distribution center, but in practice the distribution center will have a certain amount of inventory. Besides, the established model is only applicable to a simple one-to-many logistics system and does not involve other suppliers. In practice, the situation of multiple suppliers not only exists in large numbers, but also there is competition and cooperation between them. These issues require further research.

\section{REFERENCES}

1. Federgruen A, Zipkin P. H. A combined vehicle routing and inventory allocation problem $[\mathrm{J}]$. Operations Research, 1984, 32(5): 1019- 1037.

2. Burns L D, Hall R W. Distribution Strategies that Minimize Transportation and Inventory Costs[J]. Operation Research.1985.33(3):469-490.

3. Anily. S, Federguen A. One warehouse retailer systems with vehicle routing costs[J]. Management

4. Ernst R, Pyke D F. Optimal Base Stock Policies and truck capacity in a two-echelon system[J]. Naval Research Logistics. 1995.40:879-903.

5. Chandra P, Fisher L. Coordination of production and distribution planning $[\mathrm{J}]$. European Journal of Operational Research.1997.7:503-517.

Speranza M G, Ukovich W. Minimizing transportation and inventory costs for several products on a single link[J]. Operations Research.1997.42:879-894. 